CLNS 99/1634

CLEO 99-12

\title{
Observation of Radiative Leptonic Decay of the Tau Lepton
}

\author{
CLEO Collaboration
}

(June 28, 2018)

\begin{abstract}
Using $4.68 \mathrm{fb}^{-1}$ of $e^{+} e^{-}$annihilation data collected with the CLEO II detector at the Cornell Electron Storage Ring (CESR) we have studied $\tau$ radiative decays $\tau^{-} \rightarrow \nu_{\tau} \mu^{-} \bar{\nu}_{\mu} \gamma$ and $\tau^{-} \rightarrow \nu_{\tau} e^{-} \bar{\nu}_{e} \gamma$. For a $10 \mathrm{MeV}$ minimum photon energy in the $\tau$ rest frame, the branching fraction for radiative $\tau$ decay to a muon or electron is measured to be $(3.61 \pm 0.16 \pm 0.35) \times 10^{-3}$ or $(1.75 \pm$ $0.06 \pm 0.17) \times 10^{-2}$, respectively. The branching fractions are in agreement with Standard Model theoretical predictions.
\end{abstract}


T. Bergfeld, ${ }^{1}$ B. I. Eisenstein,${ }^{1}$ J. Ernst,${ }^{1}$ G. E. Gladding, ${ }^{1}$ G. D. Gollin, ${ }^{1}$ R. M. Hans,${ }^{1}$ E. Johnson ${ }^{1}$ I. Karliner,${ }^{1}$ M. A. Marsh,${ }^{1}$ M. Palmer,${ }^{1}$ C. Plager,${ }^{1}$ C. Sedlack, ${ }^{1}$ M. Selen, ${ }^{1}$ J. J. Thaler, ${ }^{1}$ J. Williams, ${ }^{1}$ K. W. Edwards, ${ }^{2}$ R. Janicek,${ }^{3}$ P. M. Patel, ${ }^{3}$ A. J. Sadoff, ${ }^{4}$ R. Ammar, ${ }^{5}$ P. Baringer,${ }^{5}$ A. Bean,${ }^{5}$ D. Besson, ${ }^{5}$ R. Davis,${ }^{5}$ S. Kotov, ${ }^{5}$ I. Kravchenko, ${ }^{5}$ N. Kwak,${ }^{5}$ X. Zhao ${ }^{5}$ S. Anderson,${ }^{6}$ V. V. Frolov,${ }^{6}$ Y. Kubota,${ }^{6}$ S. J. Lee ${ }^{6}$ R. Mahapatra,${ }^{6}$ J. J. O'Neill, ${ }^{6}$ R. Poling, ${ }^{6}$ T. Riehle, ${ }^{6}$ A. Smith, ${ }^{6}$ S. Ahmed,${ }^{7}$ M. S. Alam, ${ }^{7}$ S. B. Athar, ${ }^{7}$ L. Jian, ${ }^{7}$ L. Ling, ${ }^{7}$ A. H. Mahmood, ${ }^{7}$, M. Saleem, ${ }^{7}$ S. Timm,${ }^{7}$ F. Wappler, ${ }^{7}$ A. Anastassov, ${ }^{8}$ J. E. Duboscq, ${ }^{8}$ K. K. Gan, ${ }^{8}$ C. Gwon, ${ }^{8}$ T. Hart,${ }^{8}$ K. Honscheid, ${ }^{8}$ H. Kagan, ${ }^{8}$ R. Kass,${ }^{8}$ J. Lorenc, ${ }^{8}$ H. Schwarthoff, ${ }^{8}$ E. von Toerne,${ }^{8}$ M. M. Zoeller,${ }^{8}$ S. J. Richichi,${ }^{9}$ H. Severini, ${ }^{9}$ P. Skubic, ${ }^{9}$ A. Undrus,${ }^{9}$ M. Bishai, ${ }^{10}$ S. Chen, ${ }^{10}$ J. Fast,${ }^{10}$ J. W. Hinson, ${ }^{10}$ J. Lee, ${ }^{10}$

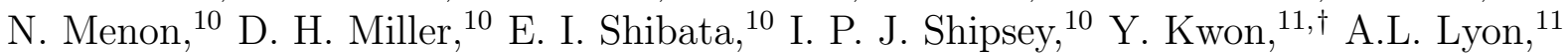
E. H. Thorndike, ${ }^{11}$ C. P. Jessop,${ }^{12}$ K. Lingel, ${ }^{12}$ H. Marsiske, ${ }^{12}$ M. L. Perl, ${ }^{12}$ V. Savinov,${ }^{12}$ D. Ugolini, ${ }^{12}$ X. Zhou, ${ }^{12}$ T. E. Coan, ${ }^{13}$ V. Fadeyev, ${ }^{13}$ I. Korolkov, ${ }^{13}$ Y. Maravin,,${ }^{13}$ I. Narsky, ${ }^{13}$ R. Stroynowski, ${ }^{13}$ J. Ye, ${ }^{13}$ T. Wlodek,,${ }^{13}$ M. Artuso, ${ }^{14}$ R. Ayad,${ }^{14}$ E. Dambasuren, ${ }^{14}$ S. Kopp,${ }^{14}$ G. Majumder, ${ }^{14}$ G. C. Moneti, ${ }^{14}$ R. Mountain,${ }^{14}$ S. Schuh,${ }^{14}$ T. Skwarnicki, ${ }^{14}$ S. Stone, ${ }^{14}$ A. Titov,${ }^{14}$ G. Viehhauser, ${ }^{14}$ J.C. Wang, ${ }^{14}$ A. Wolf, ${ }^{14}$ J. Wu, ${ }^{14}$ S. E. Csorna, ${ }^{15}$ K. W. McLean, ${ }^{15}$ S. Marka, ${ }^{15}$ Z. Xu, ${ }^{15}$ R. Godang, ${ }^{16}$ K. Kinoshita, ${ }^{16}$, I. C. Lai ${ }^{16}$ S. Schrenk, ${ }^{16}$ G. Bonvicini, ${ }^{17}$ D. Cinabro, ${ }^{17}$ R. Greene, ${ }^{17}$ L. P. Perera,${ }^{17}$ G. J. Zhou, ${ }^{17}$ S. Chan, ${ }^{18}$ G. Eigen, ${ }^{18}$ E. Lipeles, ${ }^{18}$ M. Schmidtler, ${ }^{18}$ A. Shapiro, ${ }^{18}$ W. M. Sun, ${ }^{18}$ J. Urheim, ${ }^{18}$ A. J. Weinstein,${ }^{18}$ F. Würthwein,${ }^{18}$ D. E. Jaffe, ${ }^{19}$ G. Masek, ${ }^{19}$ H. P. Paar ${ }^{19}$ E. M. Potter, ${ }^{19}$ S. Prell, ${ }^{19}$ V. Sharma,${ }^{19}$ D. M. Asner,${ }^{20}$ A. Eppich,,${ }^{20}$ J. Gronberg, ${ }^{20}$ T. S. Hill, ${ }^{20}$ D. J. Lange, ${ }^{20}$ R. J. Morrison, ${ }^{20}$ T. K. Nelson, ${ }^{20}$ R. A. Briere,${ }^{21}$ B. H. Behrens, ${ }^{22}$ W. T. Ford ${ }^{22}$ A. Gritsan, ${ }^{22}$ H. Krieg, ${ }^{22}$ J. Roy, ${ }^{22}$ J. G. Smith, ${ }^{22}$

J. P. Alexander, ${ }^{23}$ R. Baker,${ }^{23}$ C. Bebek,${ }^{23}$ B. E. Berger,${ }^{23}$ K. Berkelman,${ }^{23}$ F. Blanc, ${ }^{23}$ V. Boisvert, ${ }^{23}$ D. G. Cassel, ${ }^{23}$ M. Dickson, ${ }^{23}$ P. S. Drell, ${ }^{23}$ K. M. Ecklund,${ }^{23}$ R. Ehrlich, ${ }^{23}$ A. D. Foland, ${ }^{23}$ P. Gaidarev, ${ }^{23}$ R. S. Galik, ${ }^{23}$ L. Gibbons, ${ }^{23}$ B. Gittelman, ${ }^{23}$ S. W. Gray, ${ }^{23}$ D. L. Hartill, ${ }^{23}$ B. K. Heltsley, ${ }^{23}$ P. I. Hopman, ${ }^{23}$ C. D. Jones,${ }^{23}$ D. L. Kreinick, ${ }^{23}$ T. Lee, ${ }^{23}$ Y. Liu ${ }^{23}$ T. O. Meyer ${ }^{23}$ N. B. Mistry, ${ }^{23}$ C. R. Ng, ${ }^{23}$ E. Nordberg, ${ }^{23}$ J. R. Patterson, ${ }^{23}$ D. Peterson, ${ }^{23}$ D. Riley, ${ }^{23}$ J. G. Thayer, ${ }^{23}$ P. G. Thies,${ }^{23}$ B. Valant-Spaight,${ }^{23}$ A. Warburton, ${ }^{23}$ P. Avery, ${ }^{24}$ M. Lohner, ${ }^{24}$ C. Prescott, ${ }^{24}$ A. I. Rubiera, ${ }^{24}$ J. Yelton, ${ }^{24}$ J. Zheng, ${ }^{24}$ G. Brandenburg, ${ }^{25}$ A. Ershov ${ }^{25}$ Y. S. Gao, ${ }^{25}$ D. Y.-J. Kim,${ }^{25}$ R. Wilson, ${ }^{25}$ T. E. Browder, ${ }^{26}$ Y. Li, ${ }^{26}$ J. L. Rodriguez, ${ }^{26}$ and H. Yamamoto ${ }^{26}$

\footnotetext{
${ }^{1}$ University of Illinois, Urbana-Champaign, Illinois 61801

${ }^{2}$ Carleton University, Ottawa, Ontario, Canada K1S 5B6 and the Institute of Particle Physics, Canada

${ }^{3}$ McGill University, Montréal, Québec, Canada H3A 2T8 and the Institute of Particle Physics, Canada
}

\footnotetext{
*Permanent address: University of Texas - Pan American, Edinburg TX 78539.

${ }^{\dagger}$ Permanent address: Yonsei University, Seoul 120-749, Korea.

${ }^{\ddagger}$ Permanent address: University of Cincinnati, Cincinnati OH 45221
} 
${ }^{4}$ Ithaca College, Ithaca, New York 14850

${ }^{5}$ University of Kansas, Lawrence, Kansas 66045

${ }^{6}$ University of Minnesota, Minneapolis, Minnesota 55455

${ }^{7}$ State University of New York at Albany, Albany, New York 12222

${ }^{8}$ Ohio State University, Columbus, Ohio 43210

${ }^{9}$ University of Oklahoma, Norman, Oklahoma 73019

${ }^{10}$ Purdue University, West Lafayette, Indiana 47907

${ }^{11}$ University of Rochester, Rochester, New York 14627

${ }^{12}$ Stanford Linear Accelerator Center, Stanford University, Stanford, California 94309

${ }^{13}$ Southern Methodist University, Dallas, Texas 75275

${ }^{14}$ Syracuse University, Syracuse, New York 13244

${ }^{15}$ Vanderbilt University, Nashville, Tennessee 37235

${ }^{16}$ Virginia Polytechnic Institute and State University, Blacksburg, Virginia 24061

${ }^{17}$ Wayne State University, Detroit, Michigan 48202

${ }^{18}$ California Institute of Technology, Pasadena, California 91125

${ }^{19}$ University of California, San Diego, La Jolla, California 92093

${ }^{20}$ University of California, Santa Barbara, California 93106

${ }^{21}$ Carnegie Mellon University, Pittsburgh, Pennsylvania 15213

${ }^{22}$ University of Colorado, Boulder, Colorado 80309-0390

${ }^{23}$ Cornell University, Ithaca, New York 14853

${ }^{24}$ University of Florida, Gainesville, Florida 32611

${ }^{25}$ Harvard University, Cambridge, Massachusetts 02138

${ }^{26}$ University of Hawaii at Manoa, Honolulu, Hawaii 96822 
Unconventional models for $\tau$ decay could lead to behavior inconsistent with the Standard Model in radiative $\tau$ decay [1]. In one model $\tau$ decay occurs not only through the known s-channel exchange of a W-boson, but also through the s-channel exchange of an unknown $\mathrm{X}$ boson. In another model, $\tau$ decay occurs only through the exchange of the W-boson but the $\tau-\nu_{\tau}-\mathrm{W}$ vertex has anomalous radiative properties. In both cases, the radiative decay behavior of the $\tau$ should be altered with respect to the Standard Model expectation.

The data sample used in this work was acquired from $e^{+} e^{-}$collisions at a center-of-mass energy of $E_{c m}=2 \times E_{\text {beam }} \approx 10.6 \mathrm{GeV}$ with the CLEO II detector [2] at the Cornell Electron Storage Ring (CESR). The total integrated luminosity of the data is $4.68 \mathrm{fb}^{-1}$, corresponding to $N_{\tau \tau}=4.3 \times 10^{6} \tau$ pairs. We search for $\tau^{-} \rightarrow \nu_{\tau} \ell^{-} \bar{\nu}_{\ell} \gamma(\ell=e$ or $\mu)$ using the observed two-charged-track $\tau$ pair final states with a photon in the lepton hemisphere as defined by the plane perpendicular to the thrust axis [3]: $e^{+}+\mu^{-} \gamma, \mu^{+}+e^{-} \gamma, h^{+}+\mu^{-} \gamma$, $h^{+} \pi^{0}+\mu^{-} \gamma, h^{+}+e^{-} \gamma$, and $h^{+} \pi^{0}+e^{-} \gamma$, where $h^{+}$is a charged pion or kaon.円 The $\tau^{+}$decay products are used to tag the events.

We select events with exactly two oppositely charged tracks with scaled momentum, $x_{ \pm}=p_{ \pm} / E_{\text {beam }}$, satisfying $x_{ \pm}<0.9$ and with the angle between the two tracks greater than $90^{\circ}$. We require exactly one charged track in each hemisphere. To suppress beam-gas interactions, the distance of closest approach of each track to the interaction point must be within $0.5 \mathrm{~cm}$ transverse to the beam direction, and $5 \mathrm{~cm}$ along it. Hadronic background is suppressed by requiring the total invariant mass of particles in each hemisphere to be less than the $\tau$ mass. In computing the invariant mass, we assign the pion mass to the charged hadron. We require the two-track acollinearity in azimuth, $\xi=|| \phi_{+}-\phi_{-}|-\pi|$ where $\phi_{+}\left(\phi_{-}\right)$is the azimuthal angle of the positively (negatively) charged track, to satisfy $0.05<\xi<1.5$. The scaled missing momentum transverse to the beam, $x_{t}=p_{t} / E_{\text {beam }}$, and the angle of the missing momentum with regard to the beam line, $\theta_{\text {miss }}$, must satisfy $x_{t}>0.1$ and $\left|\cos \theta_{\text {miss }}\right|<0.8$ for all non- $h^{+} \pi^{0}$ tag modes; for the two $h^{+} \pi^{0}$-tag modes, only $x_{t}>0.05$ is required. These criteria effectively reduce potential contamination from non- $\tau$ QED events.

Photons are defined as clusters in the calorimeter with energy $E_{\gamma}>50 \mathrm{MeV}$ for $|\cos \theta|<$ 0.71 , or $100 \mathrm{MeV}$ when $0.71<|\cos \theta|<0.95$ where $\theta$ is the polar angle with respect to the beam axis. They are further required to pass a lateral shower shape requirement, that is $99 \%$ efficient for isolated photons. No charged particle track can point to within $8 \mathrm{~cm}$ of a crystal used in the energy cluster. In the signal lepton hemisphere, we require that there be only one photon, and this photon must be in the region $|\cos \theta|<0.71$. In the tag hemisphere, if the tag is a lepton, then at most one unused photon is allowed; otherwise, at most two unused photons are allowed. Photons from $\tau$ radiative leptonic decays tend to be almost collinear with the final state lepton direction, hence we require $\cos \theta_{\mu \gamma}>0.96$ in the case of muonic decay and $\cos \theta_{e \gamma}>0.99$ in the case of electronic decay.

Identified electrons are required to have scaled momenta $x_{ \pm}>0.1$ and $|\cos \theta|<0.71$. The ratio of energy deposited in the calorimeter to track momenta for electron candidates must satisfy $E_{ \pm} / p_{ \pm}>0.85$. The drift chamber specific-ionization $(d E / d x)$ for electron candidates

\footnotetext{
${ }^{1}$ Charge conjugate states are included in this analysis.
} 
must be no lower than two standard deviations below that expected for an electron. To exclude events in which a photon hides in the track's calorimeter shower, the criteria further require $E_{ \pm} / p_{ \pm}<1.1$. Muon criteria demand that the track has $|\cos \theta|<0.71$ and deposit $E_{ \pm}<0.3 \mathrm{GeV}$ in the calorimeter, consistent with a minimum-ionizing particle, and that there be hits in the muon detection system matched to the projected trajectory of the track. A muon candidate must also penetrate at least three hadronic interaction lengths for $p_{ \pm}<2.0$ $\mathrm{GeV} / c$ and five interaction lengths for $p_{ \pm}>2.0 \mathrm{GeV} / c$, corresponding to the first and second superlayers of the muon chambers. The tag $h$ is operationally defined as a charged track not identified as a lepton, with $p_{ \pm}>0.5 \mathrm{GeV} / c$ and $|\cos \theta|<0.90$. The $h \pi^{0}$ tag is defined as a reconstructed $\pi^{0}$ plus a charged track not identified as a lepton, and the charged track must satisfy $p_{ \pm}>0.3 \mathrm{GeV} / c$ and $|\cos \theta|<0.90$. A $\pi^{0}$ is reconstructed using two showers in the tag hemisphere that satisfy the photon criteria, except that only one of the showers is required to meet the lateral shower shape requirement. We require that the invariant mass of the two photons satisfy $120<m_{\gamma \gamma}<145 \mathrm{MeV}$. We exclude events in which an extra $\pi^{0}$ is found.

Additional criteria are applied to suppress mode-specific backgrounds. To reduce contamination from radiative QED processes $e^{+} e^{-} \rightarrow e^{+} e^{-} \gamma$ and $\mu^{+} \mu^{-} \gamma$, the total energy of an event must satisfy $E_{t o t}<7.5 \mathrm{GeV}$ for $h^{+}+\mu \gamma$ and $h^{+}+e \gamma$ modes. In the $h^{+}+e \gamma$ mode, to further reduce background from $e^{+} e^{-} \rightarrow e^{+} e^{-} e^{+} e^{-}(\gamma)$ we require $E_{\text {tot }}>2.8 \mathrm{GeV}$ and the $h^{+}$to satisfy $|\cos \theta|<0.71$. In the three tag modes with the $\tau^{-}$radiatively decaying to electron, in order to reduce significant background from external bremsstrahlung, we require the distance of closest approach of the electron's track to the interaction point to be within $0.08 \mathrm{~cm}$ transverse to the beam. We further require the distance between the photon candidate shower and the electron shower in the calorimeter to be greater than $25 \mathrm{~cm}$, in order to separate occasionally overlapping showers.

The detection efficiencies and backgrounds are investigated with a Monte Carlo technique. We use the KORALB/TAUOLA [4] and PHOTOS [5] MC packages to model the production and decay of $\tau$ pairs. The detector response is simulated using the GEANT program [6]. Generic Monte Carlo-produced $\tau$-pair decay events are used to study the kinematic distributions of the signal candidates and the backgrounds from $\tau$-pair decay sources. The $\cos \theta_{\ell \gamma}$ and $E_{\gamma}$ distributions from selected events for both muonic and electronic decays are shown in Fig. 1. The figure shows that data and luminosity normalized Monte Carlo expectation agree well. The small apparent disagreement at low photon energy is caused by a small relative inefficiency in the Monte Carlo reconstruction of low energy photons near muons, and is accounted for in the systematic error estimation. Using Monte Carlo-produced $\tau$ pairs in which one $\tau$ decays radiatively into a lepton and neutrinos and the another $\tau$ decays generically, we determine the total detection efficiencies to be $(3.28 \pm 0.06) \%$ for radiative muonic decay and $(2.02 \pm 0.03) \%$ for radiative electronic decay.

The backgrounds from $\tau$-pair decay sources relative to signals are shown with the $\cos \theta_{\ell \gamma}$ distributions in Fig. 2. In the muonic decay case, the significant backgrounds are ISR/FSR (initial state and final state radiation), track misidentification (mostly other particles misidentified as a muon) and neutral showers faking photons. In the electronic decay case, the electron external bremsstrahlung process is the only significant background; backgrounds such as other particles misidentified as the electron are relatively small. Figure 2 also shows that a photon from $\tau$ radiative decay to a lepton tends to have a very small angle 
with respect to the final state lepton. Further from the lepton, background photons not related to the $\tau$ leptonic decay completely dominate.

We investigate possible contamination from hadronic events by using the Lund simulation [7] and find that it is negligible. We rely upon Monte Carlo simulation of $e^{+} e^{-}$to $\mu^{+} \mu^{-}(\gamma)$ [8], $e^{+} e^{-}(\gamma)$ [9:10], $e^{+} e^{-} \mu^{+} \mu^{-}$[11], $e^{+} e^{-} \pi^{+} \pi^{-}$[11], and $e^{+} e^{-} e^{+} e^{-}$[12] final states to model backgrounds from these processes. All these background sources are small except in the two $h$ tag modes. In the case of muonic radiative decay with $h$ tag, we find that the two photon process $e^{+} e^{-} \rightarrow e^{+} e^{-} \mu^{+} \mu^{-}$contributes $0.69 \%$ to the selected sample in data and the QED process $e^{+} e^{-} \rightarrow \mu^{+} \mu^{-}(\gamma)$ contributes another $0.46 \%$. In the electronic decay case, the Monte Carlo predicts $0.42 \%$ from the two-photon process $e^{+} e^{-} \rightarrow e^{+} e^{-} e^{+} e^{-}$and $0.29 \%$ from the QED process $e^{+} e^{-} \rightarrow e^{+} e^{-}(\gamma)$. As these processes are significantly suppressed by the selection criteria and their accurate normalization is difficult to verify, a total relative error of $100 \%$ will be assigned in the final systematic errors.

Branching fractions $\mathcal{B}\left(\tau^{-} \rightarrow \nu_{\tau} \mu^{-} \bar{\nu}_{\mu} \gamma\right)$ and $\mathcal{B}\left(\tau^{-} \rightarrow \nu_{\tau} e^{-} \bar{\nu}_{e} \gamma\right)$ are calculated for $E_{\gamma}>50$ $\mathrm{MeV}$ in the laboratory frame for each mode and then converted into the $\tau$ rest frame for $E_{\gamma}^{*}>10 \mathrm{MeV}$ by applying a boost factor assuming the Standard Model photon spectrum. The factor $\epsilon_{\text {boost }}$ is determined from Monte Carlo simulation to be $0.754 \pm 0.007$ for muonic radiative decay and $0.762 \pm 0.003$ for electronic radiative decay. The branching fractions from three different tags are combined using a weighted average. The measured branching fractions from data are compared with the theoretical predictions from Monte Carlo simulation. Table [ summarizes the relative results and Table [1] shows the measured absolute branching fractions.

TABLE I. Branching fractions for $\tau^{-} \rightarrow \nu_{\tau} \mu^{-} \bar{\nu}_{\mu} \gamma$ and $\tau^{-} \rightarrow \nu_{\tau} e^{-} \bar{\nu}_{e} \gamma$ relative to Standard Model Monte Carlo expectation for all tag modes and combined results for $E_{\gamma}^{*}>10 \mathrm{MeV}$. Errors are statistical only.

\begin{tabular}{lccccc}
\hline \hline & $e \operatorname{tag}$ & $\mu \operatorname{tag}$ & $h$ tag & $h \pi^{0}$ tag & Total \\
\hline$\nu_{\tau} \mu^{-} \bar{\nu}_{\mu} \gamma$ & $1.00 \pm 0.08$ & & $0.98 \pm 0.07$ & $0.96 \pm 0.07$ & $0.98 \pm 0.04$ \\
$\nu_{\tau} e^{-} \bar{\nu}_{e} \gamma$ & & $0.95 \pm 0.06$ & $0.90 \pm 0.06$ & $0.97 \pm 0.05$ & $0.94 \pm 0.03$ \\
\hline \hline
\end{tabular}

Systematic error estimates for $\tau^{-} \rightarrow \nu_{\tau} \mu^{-} \bar{\nu}_{\mu} \gamma$ and $\tau^{-} \rightarrow \nu_{\tau} e^{-} \bar{\nu}_{e} \gamma$ are shown in Table III. The errors in the table are relative to the final branching fraction. For muonic radiative decay, we estimate the error from photon reconstruction by varying the photon selection criteria and also from a separate study of $e^{+} e^{-} \rightarrow \mu^{+} \mu^{-} \gamma$ events. The trigger efficiency systematic error is obtained by a comparison of different triggers in data and Monte Carlo. We evaluate the muon misidentification systematic error by allowing a variation of the hadron to muon misidentification rate of $15 \%$ as estimated from a sample of tracks in $\tau^{+} \tau^{-}$events in which one $\tau$ decays to a lepton and the other $\tau$ decays to $h^{+} \pi^{0}$. The energy deposition of hadrons faking muons is not well modeled in the Monte Carlo; therefore, we vary the muon maximum energy requirement to obtain its associated error. The integrated luminosity of the data at CLEO is measured with a relative error of $1 \%$; this results in a relative error of $1.4 \%$ on the total number of $\tau$ pairs produced in data, assuming a theoretical error of $1 \%$ 
TABLE II. Measured branching fractions $\mathcal{B}\left(\tau^{-} \rightarrow \nu_{\tau} \mu^{-} \bar{\nu}_{\mu} \gamma\right)$ and $\mathcal{B}\left(\tau^{-} \rightarrow \nu_{\tau} e^{-} \bar{\nu}_{e} \gamma\right)$ for $E_{\gamma}^{*}>10 \mathrm{MeV}$ and theoretical predictions from the Monte Carlo simulation. For data, the first error is statistical and the second one is systematic. For Monte Carlo, the error is based on the number of events generated. Also listed is the ratio of $\mathcal{B}\left(\tau^{-} \rightarrow \nu_{\tau} e^{-} \bar{\nu}_{e} \gamma\right)$ to $\mathcal{B}\left(\tau^{-} \rightarrow \nu_{\tau} \mu^{-} \bar{\nu}_{\mu} \gamma\right)$, $\mathcal{B}_{e \gamma} / \mathcal{B}_{\mu \gamma}$.

\begin{tabular}{lcc}
\hline \hline & data & MC \\
\hline $\mathcal{B}\left(\tau^{-} \rightarrow \nu_{\tau} \mu^{-} \bar{\nu}_{\mu} \gamma\right)\left(\times 10^{-3}\right)$ & $3.61 \pm 0.16 \pm 0.35$ & $3.68 \pm 0.02$ \\
$\mathcal{B}\left(\tau^{-} \rightarrow \nu_{\tau} e^{-} \bar{\nu}_{e} \gamma\right)\left(\times 10^{-2}\right)$ & $1.75 \pm 0.06 \pm 0.17$ & $1.86 \pm 0.01$ \\
$\mathcal{B}_{e \gamma} / \mathcal{B}_{\mu \gamma}$ & $4.85 \pm 0.27 \pm 0.57$ & $5.05 \pm 0.04$ \\
\hline \hline
\end{tabular}

for the $\tau$-pair production cross section [4]. The uncertainty for the track finding efficiency is estimated from a visual scan of $e^{+} e^{-} \rightarrow e^{+} e^{-}$events selected using shower information only and a study of pion finding efficiency in $\tau^{+} \tau^{-}$events in which one $\tau$ decays to a lepton and the other $\tau$ decays to $3 \pi^{ \pm}\left(\pi^{0}\right)$. Other errors are small and we estimate these errors by either using an independent sample or by varying related individual requirements.

The largest background to the decay $\tau^{-} \rightarrow \nu_{\tau} e^{-} \bar{\nu}_{e} \gamma$ comes from electron external bremsstrahlung. This process contributes about $40 \%$ of the observed $\gamma$ 's. Its systematic error contribution is estimated from comparisons of data and Monte Carlo simulation for accepted $e^{+} e^{-} \gamma$ events from $e^{+} e^{-} \rightarrow e^{+} e^{-} e^{+} e^{-}(\gamma)$. The comparison indicates that external bremsstrahlung events in our Monte Carlo simulation are $(11 \pm 7) \%$ more likely than in data. This result is also confirmed by comparing the number of photon conversion events from $\pi^{0}$ decays between data and Monte Carlo. We estimate a propagated branching fraction error of $6.9 \%$ by allowing a variation of as much as $18 \%$ for this background. The error from photon reconstruction is estimated by varying the photon selection criteria. All remaining errors are estimated as in the muonic case. In calculating the systematic error for the ratio $\mathcal{B}_{\text {er }} / \mathcal{B}_{\mu \gamma}$, errors from the trigger, the number of $\tau$ pairs and the track-finding efficiency cancel.

There have been measurements of $\tau$ radiative muonic decay from MARK II [13] and OPAL 14. The OPAL result is more recent and more precise. OPAL reports a measurement of the branching fraction $\mathcal{B}\left(\tau^{-} \rightarrow \nu_{\tau} \mu^{-} \bar{\nu}_{\mu} \gamma\right)=(3.0 \pm 0.4 \pm 0.5) \times 10^{-3}$ for $E_{\gamma}^{*}>20 \mathrm{MeV}$. Converting our result for $E_{\gamma}^{*}>10 \mathrm{MeV}$ to a result for $E_{\gamma}^{*}>20 \mathrm{MeV}$ gives a measurement of $\mathcal{B}\left(\tau^{-} \rightarrow \nu_{\tau} \mu^{-} \bar{\nu}_{\mu} \gamma\right)=(3.04 \pm 0.14 \pm 0.30) \times 10^{-3}$, which is in excellent agreement with the OPAL result but with an error smaller by a factor of two. CLEO has previously observed $\tau$ radiative electronic decay [15], but this is the first direct measurement of the branching fraction.

As pointed out in Refs. 16, 17, Lorentz structure parameters in $\tau$ decay that are difficult to measure directly in non radiative decays can also be investigated in radiative $\tau$ decay. For example, the probability $Q_{R}^{\ell}$ of the $\tau$ decaying into a right-handed charged daughter lepton is given by $Q_{R}^{\ell}=\frac{1}{2}\left(1-\xi^{\prime}\right)\left(\xi^{\prime}=1\right.$ in the Standard Model). If we could extract the Michel type parameter $\xi^{\prime}$ by measuring the partial $\tau$ radiative decay rate [16], then $Q_{R}^{\ell}$ could be limited. However, the differential $\tau$ radiative decay rate is most sensitive to $\xi^{\prime}$ for photons emitted in the direction opposite to the daughter lepton, an area dominated by photons from 
TABLE III. Summary of systematic errors from different sources for $\tau$ muonic and electronic radiative decays.

\begin{tabular}{lcc}
\hline \hline Source & $\tau^{-} \rightarrow \nu_{\tau} \mu^{-} \bar{\nu}_{\mu} \gamma$ & $\tau^{-} \rightarrow \nu_{\tau} e^{-} \bar{\nu}_{e} \gamma$ \\
\hline external bremsstrahlung & $\approx 0.0$ & 6.9 \\
photon reconstruction & 5.9 & 4.6 \\
trigger & 5.0 & 5.0 \\
track misidentification & 4.4 & 1.1 \\
muon shower energy requirement & 3.6 & $N A$ \\
$N_{\tau \tau}$ & 1.4 & 1.4 \\
track-finding efficiency & 1.0 & 1.0 \\
non $\tau$ sources & 0.9 & 0.1 \\
ISR/FSR & 0.8 & 0.2 \\
\hline Total & $9.8 \%$ & $9.9 \%$ \\
\hline \hline
\end{tabular}

other sources. This indicates that we are unable to set useful limits using the experimental method described here.

In summary, we have performed the first measurement of $\mathcal{B}\left(\tau^{-} \rightarrow \nu_{\tau} e^{-} \bar{\nu}_{e} \gamma\right)$ and an improved measurement of $\mathcal{B}\left(\tau^{-} \rightarrow \nu_{\tau} \mu^{-} \bar{\nu}_{\mu} \gamma\right)$ using the CLEO detector at the CESR. Within the errors of the measurements we find that the magnitude of the decay rates and the kinematic distributions agree with expectations of conventional electromagnetic and weak interaction theory. We also conclude that it is not currently possible to set useful limits on the parameters proposed in [16,17] using the experimental method described in this letter.

We gratefully acknowledge the effort of the CESR staff in providing us with excellent luminosity and running conditions. This work was supported by the National Science Foundation, the U.S. Department of Energy, the Research Corporation, the Natural Sciences and Engineering Research Council of Canada, the A.P. Sloan Foundation, the Swiss National Science Foundation, and the Alexander von Humboldt Stiftung. 


\section{REFERENCES}

[1] M. L. Perl, in Proceedings of Fifth International WEIN Symposium on Physics Beyond the Standard Model, edited by P. Herczeg, C. M. Hoffman, and H. V. KlapdorKleingrothaus (World Scientific, Singapore, 1999).

[2] CLEO Collaboration, Y. Kubota et al., Nucl. Instrum. Methods Phys. Res., Sec. A 320, 66 (1992).

[3] E. Farhi, Phys. Rev. Lett. 39, 1587 (1977).

[4] S. Jadach and Z. Was, Comput. Phys. Commun. 36, 191 (1985); S. Jadach, J.H. Kuhn, and Z. Was, ibid. 64, 275 (1991); 70, 69 (1992); 76, 361 (1993).

[5] E. Barberio, B. van Eijk, and Z. Was, Comput. Phys. Commun. 79, 291 (1994).

[6] R. Brun et al., CERN Report No. CERN-DD/EE/84-1, 1987 (unpublished).

[7] T. Sjöstrand and M. Bengtsson, Compt. Phys. Commun. 43, 367 (1987).

[8] R. Kleiss and S. van der Marck, Nucl. Phys. B 342, 61 (1990).

[9] F. Berends and R. Kleiss, Nucl. Phys. B 228, 537 (1983).

[10] S. Jadach, E. Richter-Was, B.F.L. Ward, and Z. Was, Phys. Lett. B 268, 253 (1991).

[11] V. M. Budnev et al., Phys. Rep. 15, 181 (1975).

[12] J. Vermaseren, Nucl. Phys. B 229, 347 (1983).

[13] D. Y. Wu et al., Phys. Rev, D 41, 2339 (1990).

[14] OPAL Collaboration, G. Alexander et al., Phys. Lett. B 388, 437 (1996).

[15] CLEO Collaboration, D. S. Akerib et al., Phys. Rev. Lett. 69, 3610 (1992); 71, 3395 (E) (1993).

[16] S. Stahl and H. Voss, Z. Phys. C 74, 73 (1997).

[17] W. Eichenberger, R. Engfer, and A. Van Der Schaaf, Nucl. Phys. A 412, 523 (1984). 

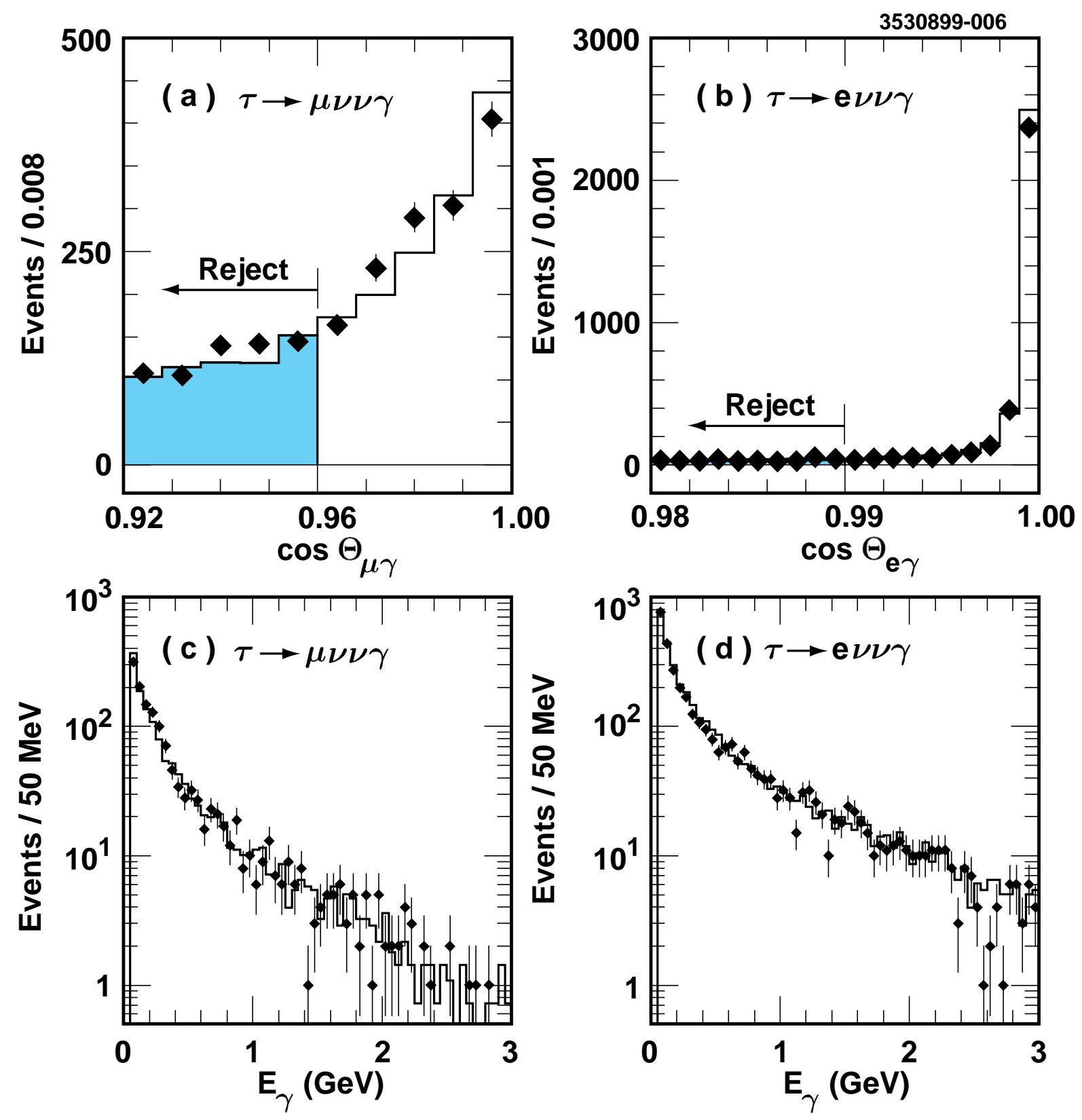

FIG. 1. Distributions in $\cos \theta_{\ell \gamma}$ and $E_{\gamma}$ for data (diamonds) and Monte Carlo (histogram) for both muonic and electronic radiative decays of the $\tau$. Each distribution shown here is the sum over all tag modes. Only events satisfying the $\cos \theta_{\ell \gamma}$ requirement are used in the $E_{\gamma}$ distributions. 


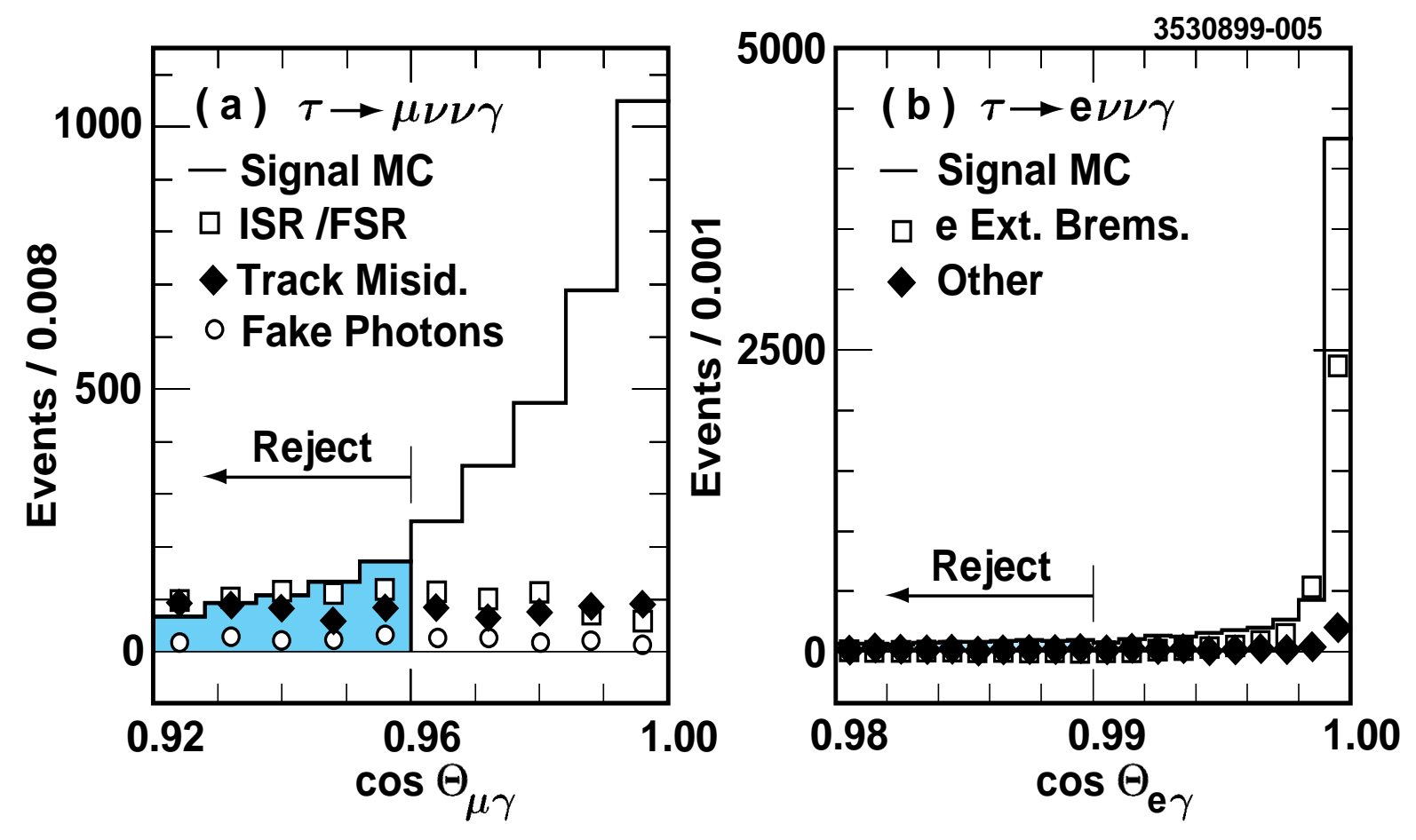

FIG. 2. Distributions in $\cos \theta_{\ell \gamma}$ for signals and different tau source backgrounds from Monte Carlo simulations. 